\title{
Mobile Schwannoma of the Cauda Equina Diagnosed by Magnetic Resonance Imaging and Magnetic Resonance Myelography: A Case Report and Review of the Literatures
}

\author{
Dong Yeob Lee ${ }^{1}$, Hoon Jae Chung ${ }^{2}$ \\ Departments of ${ }^{1}$ Neurosurgery, ${ }^{2}$ Orthopedic Surgery, Bumin Hospital Seoul, Seoul, Republic of Korea
}

Corresponding author: Dong Yeob Lee

Department of Neurosurgery, Bumin Hospital Seoul, 389,

Gonghang-daero, Gangseo-gu, Seoul 07590, Republic of Korea

Tel: +82-2-2620-0004

Fax: +82-2-2620-0167

E-mail: nsdrlee@naver.com

Received: August 25, 2017

Revised: September 19, 2017

Accepted: September 23, 2017
Here we report a 65-year-old man with a mobile intradural extramedullary schwannoma of the cauda equina. Preoperative magnetic resonance imaging demonstrated mobility of the tumor. Preoperative magnetic resonance myelography showed that tumor migration did not occur continuously. It occurred suddenly instead. With spinous process splitting laminectomy, the tumor was successfully removed.

Key Words: Cauda equina; Lumbar vertebrae; Magnetic resonance imaging; Myelography; Neurilemmoma

\section{INTRODUCTION}

Mobile intradural extramedullary (IDEM) schwannoma is a rare disease entity ${ }^{1-9-9-17)}$. Only 24 cases of mobile schwannoma have been reported in the literatures ${ }^{5-7,14,16)}$. The mobility of schwannoma might result in failure to locate the tumor during surgery $^{6,16)}$. The mobility of schwannoma has been suggested to be associated with changes in intra-thecal pressure or spinal posture $^{1,2,6,9-12,17)}$. However, its mechanism is still not clearly understood.

We report a case of mobile schwannoma of the cauda equina diagnosed by magnetic resonance (MR) imaging (MRI) and MR myelography. MRI and gadolinium-enhanced MRI performed $2 \mathrm{hr}$ later demonstrated a mobile IDEM tumor. MR myelography performed one week thereafter showed that the tumor moved suddenly in cranial direction during examination. This suggests that changes in intrathecal pressure is closely related to the mobility of the tumor. We also reviewed literatures about mobile schwannoma.

\section{CASE REPORT}

A 65-year-old man presented at our hospital suffering from pain in his lower back as well as paresthesia in both legs for 3 months prior. Physical examinations revealed absence of motor weakness. Straight leg raising test was negative. Labora- tory examinations did not reveal any abnormal findings. MRI revealed a well-demarcated ovoid lesion at $4 \mathrm{~cm}$ in size with low signal intensity at the level of L2-3 vertebral body (extending between L2 mid-body and L3 lower body) (Fig. 1A), suggesting an IDEM tumor. A gadolinium-enhanced MRI performed $2 \mathrm{hr}$ later showed that the tumor had migrated upward to the L2 body (between L1 lower endplate and L2 lower endplate) (Fig. 1B). Therefore, mobile IDEM tumor of the cauda equina was diagnosed. One week after MRI examination, the patient underwent MR myelography which revealed a large ovoid filling defect at the site of the mass. It moved suddenly in the cranial direction during examination (Fig. 2).

We performed spinous process splitting laminectomy between L1 and L3 levels ${ }^{8}$. Following incision at the dural midline, the tumor was identified and carefully dissected from surrounding roots. The tumor spontaneously extruded from the dural space with several strong cerebrospinal fluid (CSF) pulses, after which it was completely removed. After the surgery, the patient reported improvement for his back pain and paresthesia. Histological examination was consistent with characteristics of schwannoma (Fig. 3).

\section{DISCUSSION}

Since the first report of mobile spinal schwannoma by Tomimatsu et al. ${ }^{15)}$ in 1974, 25 cases (including the present case) have been reported (Table 1$)^{1-7,9-17)}$. All reported tumors were 
Table 1. Mobile schwannoma of spine (summary of the literatures)

\begin{tabular}{|c|c|c|c|c|c|c|}
\hline No & Studies & Age/Sex & Location & Discrepancy (vertebra) & Migration & Imaging modality \\
\hline 1 & Tomimatsu et al. ${ }^{15)}$ & $47 / M$ & C4-6 & 2 & Rostral & Myelography \\
\hline 2 & Hollin et al. ${ }^{2)}$ & $56 / F$ & L1 & 3 & Rostral & Myelography \\
\hline 3 & Pau et al. ${ }^{11)}$ & $50 / F$ & L2 & 1 & Rostro-caudal & Myelography \\
\hline 4 & Tavy et al. ${ }^{13)}$ & $45 / M$ & LI-2 & 2 & Caudal & Myelography \\
\hline 5 & Isu et al. ${ }^{4)}$ & $51 / F$ & $\mathrm{~T} 11-12$ & 1 & Caudal & MRI \\
\hline 6 & Isu et al. ${ }^{4)}$ & $42 / M$ & $\mathrm{~T} 12$ & 1 & Caudal & MRI \\
\hline 7 & Isu et al. ${ }^{4}$ & $52 / \mathrm{M}$ & L1 & 0.5 & Caudal & MRI \\
\hline 8 & Satoh et al. ${ }^{12)}$ & $66 / M$ & L1 & 1 & Caudal & Myelography \\
\hline 9 & Namura et al. ${ }^{10)}$ & $51 / M$ & $\mathrm{~T} 4-5$ & 5 & Rostro-caudal & MRI \& myelography \\
\hline 10 & Varughese and Mazagri ${ }^{17)}$ & $65 / M$ & $\mathrm{~L} 5$ & 1 & Rostral & Myelography \\
\hline 11 & Varughese and Mazagri ${ }^{17)}$ & 78/M & L3 & 1 & Rostral & Myelography \\
\hline 12 & lizuka et al..$^{3)}$ & $48 / \mathrm{M}$ & C7-T1 & 1 & Caudal & MRI \\
\hline 13 & Friedman et al." & $28 / M$ & L4 & 1 & Rostral & MRI \& intraoperative USG \\
\hline 14 & Friedman et al. ${ }^{1)}$ & $33 / M$ & L5 & 0.5 & Rostral & MRI \& intraoperative USG \\
\hline 15 & Friedman et al. ${ }^{1)}$ & $41 / F$ & L2 & 0.5 & Rostral & MRI \& intraoperative USG \\
\hline 16 & Marin-Sanabria et al. ${ }^{9}$ & $27 / M$ & $\mathrm{~L} 1$ & 0.5 & Caudal & MRI \& intraoperative MRI \\
\hline 17 & Marin-Sanabria et al. ${ }^{9}$ & $41 / M$ & L2-3 & 1 & Rostral & MRI \& intraoperative MRI \\
\hline 18 & Kim et al. ${ }^{6)}$ & $45 / M$ & L3-4 & 1 & Rostral & MRI \\
\hline 19 & Kim et al. ${ }^{6)}$ & $32 / M$ & T10-11 & 0.5 & Rostral & MRI \\
\hline 20 & Kim et al. ${ }^{6)}$ & $27 / M$ & L3-4 & 1 & Rostral & MRI \\
\hline 21 & Khan et al. ${ }^{5)}$ & $52 / \mathrm{M}$ & T10-11 & 3 & Rostral & MRI \\
\hline 22 & Terada et al. ${ }^{14)}$ & $68 / M$ & $\mathrm{C} 5-7$ & 1 & Caudal & MRI \& intraoperative USG \\
\hline 23 & Toscano et al. ${ }^{16)}$ & $40 / M$ & $\mathrm{~T} 11-12$ & 1 & Caudal & MRI \& intraoperative USG \\
\hline 24 & Kothari et al. ${ }^{7}$ & $70 / M$ & L2-3 & 1 & Caudal & MRI \\
\hline 25 & This study & $65 / M$ & L2-3 & 0.5 & Rostral & MRI \& MR myelography \\
\hline
\end{tabular}

M: male; F: female; MRI: magnetic resonance imaging; USG: ultrasonography; MR: magnetic resonance.

located in the IDEM area. Of the 25 cases, 21 (84\%) were males and $4(16 \%)$ were females. Their mean age was 48.8 years (range, 27-78 years). The most common place of mobile schwannoma was in the lumbar spine $(60 \%)$, followed by the thoracic $(12 \%)$, thoracolumbar (12\%), thoracocervical, and cervical spine ${ }^{6,7,16)}$. Mean migration distance was 1.28 vertebral bodies (range, 0.5-5 vertebral bodies). Migration toward rostral direction occurred in $52 \%$ of cases, which was more than that toward the caudal direction (40\%) or, the rostro-caudal direction (8\%). In the present case of a 65-year-old man, the tumor was initially located between the L2 mid-body and the L3 lower body. After migrating upward, it finally located between the L1 lower endplate and the $\mathrm{L} 2$ lower endplate. The migration distance was about 0.5 vertebral bodies.

The mobility of schwannoma has been determined using MRI, conventional myelography, intraoperative ultrasonography, and/or intraoperative MRI, to demonstrate movement of the tumor in cranial, caudal, or both directions ${ }^{1,6,7,9,15,16)}$. Repeated radiologic studies with certain time interval is essential for the diagnosis of mobile schwannoma ${ }^{6,7)}$. In the present study, the interval between initial MRI and gadolinium-enhanced MRI was $2 \mathrm{hr}$. Toscano et al. ${ }^{16)}$ also performed repeated MRI after the patient complained of new-onset of bladder incontinence, which showed tumor movement. In the present case, MR myelography showed the moment of tumor migration by chance. The tumor moved suddenly in the cranial direction, although the patient did not cough or change position during examination.

The mechanism underlying spinal schwannoma mobility has not been clearly explained yet. Changes in spinal posture have been suggested to play a role in this rare phenomenon ${ }^{11)}$. Any procedures that increases intra-abdominal, intra-thoracic, or intra-thecal pressure have been suggested to be associated with tumor mobility ${ }^{1,6,9,10,17)}$. In the present case, the patient neither coughed nor changed position during the examination. During operation, the tumor spontaneously extruded from the dural space with several strong CSF pulses. Therefore, changes in CSF dynamics due to blockage of CSF pathway by IDEM 


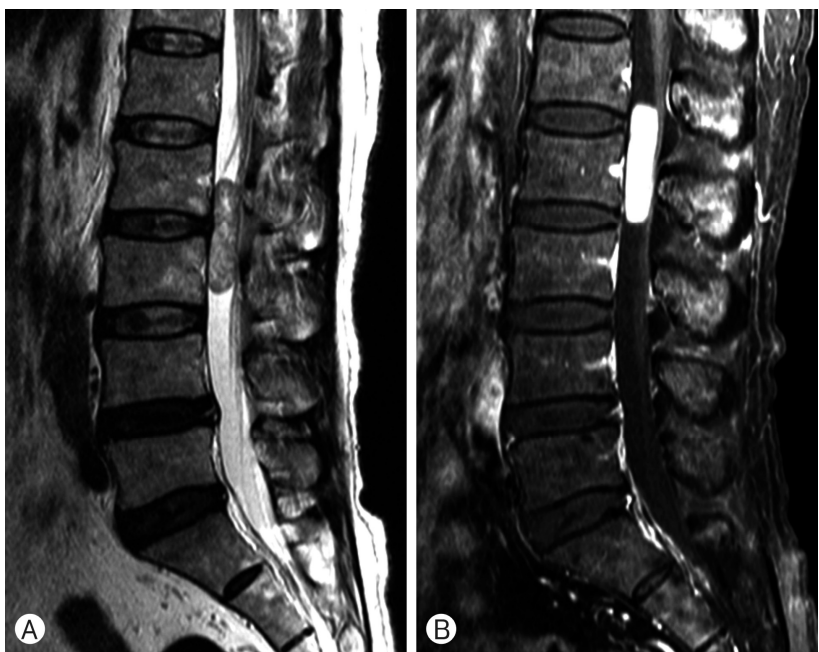

Fig. 1. T2-weighted sagittal magnetic resonance imaging (MRI) (A) demonstrating well-demarcated ovoid-shaped mass lesion at $4 \mathrm{~cm}$ in size with low-signal intensity at L2-3 level (between L2 mid-body and L3 lower body), suggesting an intradural extramedullary tumor. Gadolinium-enhanced MRI (B), taken 2 hr later showing upward-migration of the tumor to the L2 body (between L1 lower endplate and L2 lower endplate).

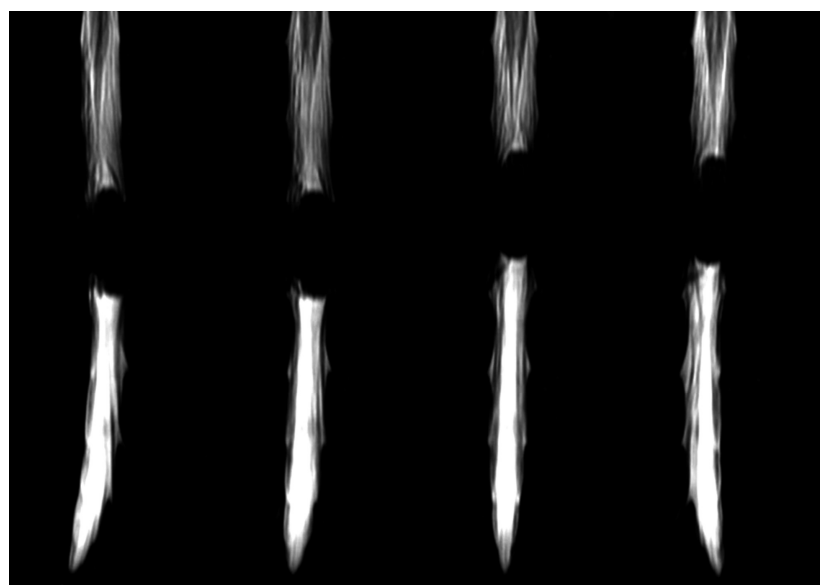

Fig. 2. Serial images of magnetic resonance (MR) myelography taken one week after MR imaging examination showing a large ovoid filling defect at the site of the mass which moved suddenly in the cranial direction during examination.

tumor might have caused tumor mobility in the present case.

Schwannoma mobility might result in failure to locate the tumor in initial operation, leading to unnecessary second operation $^{5-7,13)}$. Intraoperative myelography, ultrasonography, and MRI have been suggested as useful tools to locate mobile schwannomas ${ }^{1,9,16)}$. Intraoperative myelography was not performed in the present case because of the dye itself might cause tumor migration ${ }^{6}$. MRI and ultrasonography were also unavailable for intraoperative diagnostic use. Therefore, we removed the tumor using spinous process splitting laminectomy between L1 and L3 to expose the whole range of tumor mobility. Spinous

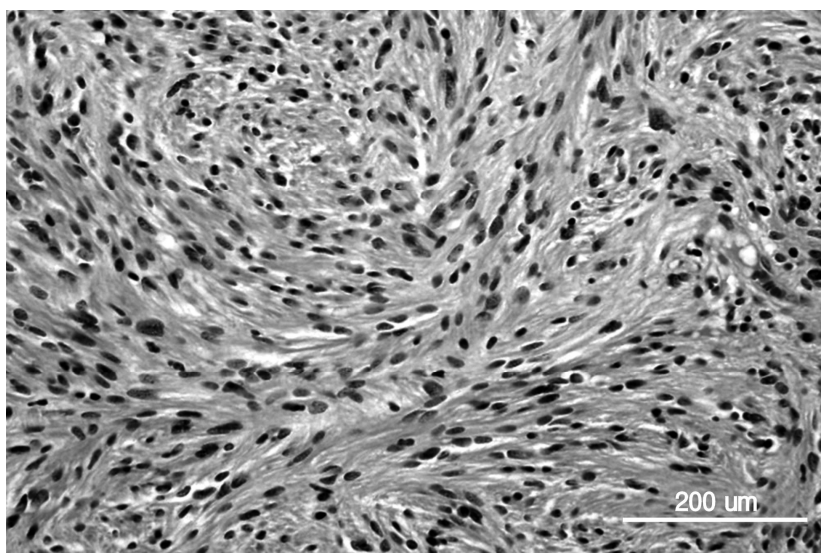

Fig. 3. Microscopic images showing compact or loose fascicles of elongated cells with mild nuclear pleomorphism (hematoxylin and eosin stain [H \& E], $\times 400$ magnification).

process splitting laminectomy is especially useful as an alternative to conventional laminectomy in spinal tumor surgery, since it allows wide exposure of the tumor while preserving posterior supporting structures ${ }^{8)}$.

\section{CONCLUSION}

A rare case of mobile IDEM schwannoma diagnosed by MRI and MR myelography is reported here. Changes in CSF dynamics due to blockage of CSF pathway by IDEM tumor might have caused tumor mobility in the present case. The tumor was successfully removed by spinous process splitting laminectomy.

\section{CONFLICTS OF INTEREST}

No potential conflict of interest relevant to this article was reported.

\section{REFERENCES}

1. Friedman JA, Wetjen NM, Atkinson JL: Utility of intraoperative ultrasound for tumors of the cauda equina. Spine (Phila $\mathrm{Pa}$ 1976) 28:288-290, 2003

2. Hollin SA, Drapkin AJ, Wancier J, Huang YP: Mobile schwannoma of the cauda equina. Case report. J Neurosurg 48:135137, 1978

3. Iizuka $\mathrm{H}$, lida T, Akai T: Mobile neurinoma of the cervicothoracic junction. Surg Neurol 50:492-493, 1998

4. Isu T, Iwasaki Y, Akino M, Nagashima M, Abe H: Mobile schwannoma of the cauda equina diagnosed by magnetic resonance imaging. Neurosurgery 25:968-971, 1989

5. Khan RA, Rahman A, Bhandari PB, Khan SI: Double migration of a schwannoma of thoracic spine. BMJ Case Rep [epub ahead of print, 2013. doi: 10.1136/bcr-2012-008182]

6. Kim SB, Kim HS, Jang JS, Lee SH: Mobility of intradural 
extramedullary schwannoma at spine : report of three cases with literature review. J Korean Neurosurg Soc 47:64-67, 2010

7. Kothari A, Singh N, Anjum R: Mobile Schwannomas of lumbar spine: A diagnostic dilemma. J Clin Orthop Trauma 8:197-200, 2017

8. Lee DY, Lee SH: Spinous process splitting laminectomy for lumbar canal stenosis: a critical appraisal. Minim Invasive Neurosurg 51:204-207, 2008

9. Marin-Sanabria EA, Sih IM, Tan KK, Tan JS: Mobile cauda equina schwannomas. Singapore Med J 48:e53-e56, 2007

10. Namura S, Hanakita J, Suwa H, Mizuno M, Ohtsuka T, Asahi M: Thoracic mobile neurinoma. Case report. J Neurosurg 79: 277-279, 1993

11. Pau A, Orunesu G, Sehrbundt Viale E, Turtas S, Zirattu G: Mobile neurinoma of the cauda equina. Case report. Acta Neurochir (Wien) 60:115-117, 1982
12. Satoh T, Kageyama T, Kamata I, Date I: Mobile neurinoma of the cauda equina; a case report. No Shinkei Geka 19:891-896, 1991

13. Tavy DL, Kuiters RR, Koster PA, Hekster RE: Elusive tumor of the cauda equina. Case report. J Neurosurg 66:131-133, 1987

14. Terada Y, Toda H, Yokote A, Iwasaki K: A mobile schwannoma of the cervical spinal cord: case report and review of the literature. Neurosurgery 78:E156-E159, 2016

15. Tomimatsu T, Yanamura I, Kawaguchi N, Fujii K, Isobe Y: A mobile tumor of the cervical spinal cord. A case report. Kantoh Seisaisi 5:213?218, 1974

16. Toscano DT, Felbaum DR, Ryan JE, Sayah A, Nair MN: Mobile schwannoma of the lumbar spine: a case report and review of the literature. Cureus 8:e715, 2016

17. Varughese G, Mazagri R: Mobile tumours in the lumbar spinal canal: a diagnostic problem. Can J Surg 40:59-63, 1997 